\title{
Pourquoi le riz étuvé local est-il peu disponible sur les marchés urbains du Bénin?
}

\author{
Laurenda Todomé ${ }^{1, *}$, Caroline Lejars ${ }^{1,2,4}$, Frédéric Lançon ${ }^{3,5}$ et Rachid Hamimaz ${ }^{1}$ \\ ${ }^{1}$ Institut Agronomique et Vétérinaire Hassan II, Rabat, Maroc \\ 2 CIRAD, UMR G-EAU, 34398 Montpellier, France \\ ${ }^{3}$ CIRAD, UMR ART-DEV, 34398 Montpellier, France \\ ${ }^{4}$ G-EAU, Univ Montpellier, Montpellier, France \\ 5 ART-DEV, Univ Montpellier, Montpellier, France
}

\begin{abstract}
Résumé - Le riz étuvé local, par ses valeurs nutritionnelles meilleures que celles du riz blanc, est une alternative intéressante pour infléchir la dépendance du Bénin au riz blanc importé. Depuis la crise rizicole de 2008, des efforts ont été consentis pour augmenter la production, améliorer la qualité et faciliter l'accès du riz étuvé local aux marchés urbains. Pourtant, en 2016, ce riz reste quasi introuvable sur les marchés de Cotonou, principale ville du Bénin. Conduit sur un échantillon de 210 acteurs, cet article utilise le paradigme StructureComportement-Performance pour identifier les principales raisons de l'indisponibilité du riz étuvé local sur les marchés de Cotonou. Nos résultats indiquent que le riz étuvé local ne représente que $3 \%$ de l'offre de riz, soit cinq fois moins que le riz étuvé importé. La méconnaissance des valeurs nutritionnelles du rizétuvé local par les ménages, son prix supérieur à celui du riz étuvé importé et son indisponibilité tout au long de l'année et chez tous les commerçants sont les principales raisons de la faible demande. Avec des sensibilisations (séances de sensibilisation, des panneaux et spots publicitaires) sur les valeurs nutritionnelles du riz étuvé local, les consommateurs urbains pourraient modifier leur comportement en faveur du riz étuvé local. Cependant, étant donné que le riz étuvé est également importé, nos résultats suggèrent qu'une étude plus fine soit réalisée sur les critères de choix du riz étuvé afin d'éviter la dépendance du Bénin au riz étuvé importé.
\end{abstract}

Mots clés : dépendance riz importé / consommateurs urbains / riz étuvé local / Cotonou

\begin{abstract}
Why is local parboiled rice unavailable in urban markets in Benin? Local parboiled rice, because of its nutritional values better than white rice, is an interesting alternative to reduce Benin's dependence on imported white rice. Since the rice crisis of 2008 , Benin has improved the production and the quality of local parboiled rice to facilitate its access to urban markets. Despite these efforts, local parboiled rice is not available on Cotonou's markets, the first town of Benin. Based on a sample of 210 actors, this article uses the paradigm S-C-P (Structure-Behavior-Performance) to identify the main reasons for the unavailability of this rice in Cotonou's markets. Our results show that local parboiled rice represents $3 \%$ of rice supply of Cotonou, five times less than imported parboiled rice. The demand for local parboiled rice is low due to consumers' ignorance of its nutritional values, higher prices than imported parboiled rice and unavailability throughout the year among all traders by households. Awareness raising efforts (awareness sessions, billboards and advertising spots) on the nutritional values of local parboiled rice could change urban consumers' behavior in favor of local parboiled rice. However, parboiled rice is also imported, so we recommend to study and analyze the determinants of parboiled rice choice in order to avoid Benin's dependence on imported parboiled rice.
\end{abstract}

Keywords: rice import dependance / urban consumers / local parboiled rice / Cotonou

\section{Introduction}

Depuis trois décennies, l'approvisionnement alimentaire des villes d'Afrique de l'Ouest est une préoccupation majeure

\footnotetext{
*Auteur de correspondance : laurendatodome@gmail.com
}

pour la sécurité alimentaire de la région et pour l'équilibre alimentaire mondial. La libéralisation dans les années 1990 et la forte urbanisation ont amené les pouvoirs publics en place à prendre l'option de nourrir leurs villes à partir de céréales importées à des coûts inférieurs aux produits locaux (Hatcheu, 2003). Importer du riz était plus économique que d'en produire 
et permettait d'assurer une sécurité alimentaire urbaine, sans dépendre d'une agriculture vivrière locale incapable de répondre à la forte croissance urbaine (Lançon, 2011). En raison de la facilité et la rapidité de sa préparation, le riz est progressivement entré dans les habitudes alimentaires et est devenu la céréale préférée des populations urbaines en Afrique de l'Ouest (Tomlins et al., 2005).

Au Bénin, le riz est la deuxième céréale la plus consommée (Adégbola et al., 2011). C'est un aliment ordinaire consommé par les ménages et dans les restaurations collectives sous plusieurs formes (Kinkpé et al., 2016). Sa consommation est passée de $10 \mathrm{~kg} / \mathrm{habitant} / \mathrm{an}(\mathrm{kg} / \mathrm{hbt} / \mathrm{an})$ en 1990 à $67 \mathrm{~kg} / \mathrm{hbt} / \mathrm{an}$ en 2010 et les importations de riz ont été multipliées par 5 pendant la même période (Demont et al., 2017). Les efforts de développement de la riziculture locale ont induit une augmentation de la production locale et une amélioration de la qualité du riz local avec la mise en place d'unités de transformation et de kits d'étuvage modernes (Konnon et al., 2014). Malgré ces efforts, le taux de dépendance du Bénin au riz importé est de $75 \%$ en 2015 (Demont et al., 2017) et le riz local reste peu disponible sur les marchés urbains (Konnon et al., 2014).

Plusieurs auteurs démontrent que les consommateurs béninois ont une préférence pour le riz blanc importé, et expliquent que le riz blanc local n'arrive pas à concurrencer le riz blanc importé à cause de sa qualité jugée « inférieure » par les consommateurs urbains (Kinkpé et al., 2016; Demont et al., 2017). Cette qualité « inférieure» du riz blanc local, due aux pratiques post-récolte peu appropriées utilisées par les riziculteurs et les transformateurs, peut être améliorée grâce à l'étuvage. En effet, l'étuvage, qui consiste à ré-humidifier, chauffer et sécher le paddy avant le décorticage, permet d'obtenir un riz de qualité physique, organoleptique et nutritionnelle supérieure à celle du riz blanc (Houssou et al., 2016; Ndindeng et al., 2015).

En 2012, une étude réalisée à Cotonou a montré que les ménages interrogés, après avoir reçu des informations sur les valeurs nutritionnelles du riz étuvé local, ont acheté ce riz au détriment de leur riz préféré, le riz blanc importé (Demont et al., 2013). Le riz étuvé local, par ses valeurs nutritionnelles meilleures que celles du riz blanc, pourrait donc être une alternative intéressante pour infléchir la dépendance du Bénin au riz importé. Mais trois ans après l'étude de Demont et al. (2013), le riz étuvé local est pourtant quasi introuvable sur les marchés de Cotonou.

Le présent article étudie les raisons de l'indisponibilité du riz étuvé local sur les marchés de Cotonou.

\section{Méthodologie}

\subsection{Cadre analytique de l'étude}

L'étude s'est appuyée sur le paradigme StructureComportement-Performance (SCP), un outil d'analyse des systèmes de commercialisation des marchés agricoles. Cet outil a été utilisé pour analyser l'efficacité des marchés du gari au Nigéria (Olagunju et al., 2012), du maïs (Lutz, 1994), du porc (Adegbidi, 1996) et du riz (Gounsè, 2006) au Bénin. Le SCP repose sur trois éléments indissociables : la structure du marché, la conduite des acteurs et la performance du marché.
La structure du marché concerne l'ensemble des caractéristiques physiques et organisationnelles du marché qui influencent et impactent la nature des transactions entre les acteurs dudit marché. Dans notre étude, les recherches sont orientées sur les acteurs du marché et les types de riz rencontrés.

La conduite des acteurs se réfère à la stratégie et aux comportements individuels ou collectifs des différents acteurs (du producteur au consommateur) pour s'adapter aux variations des marchés et maximiser leur profit. Dans cette étude, nous mettons l'accent sur les stratégies d'approvisionnement, le mode de paiement et les relations entre les acteurs (producteurs, étuveuses, commerçants et consommateurs).

La performance du marché est l'évaluation économique de la structure et de la conduite du marché. Elle s'intéresse aux relations entre les marges et les coûts (Olagunju et al., 2012). Dans cet article, en plus des coûts engagés par les acteurs et des marges obtenues, une analyse comparative de la rentabilité de la vente au détail du riz étuvé (local versus importé) a été faite. Les indicateurs utilisés sont la Marge nette en filière (MN) et le Retour sur investissement (RSI), deux indicateurs utilisés dans le diagnostic financier des filières agricoles (Dabat et al., 2007). Ils sont calculés selon les formules suivantes :

$$
M N=R P-C T,
$$

$R S I=100 \times \frac{M N}{C T}$,où $R P$ représente la recette obtenue par l'acteur pour la vente du bien $\mathrm{P}$ et $C T$, le coût total de production du bien $\mathrm{P}$ (y compris les taxes).

\subsection{Période et outils de collecte}

Les données de l'étude ont été collectées de juillet 2015 à février 2016. Des questionnaires semi-structurés, avec des questions à choix multiples (producteurs et étuveuses) et des questions ouvertes (consommateurs) ont été combinés à l'observation pour collecter les données.

\subsection{Zone d'étude}

La figure 1 présente les deux zones d'étude. Les données relatives à la commercialisation et à la consommation du riz étuvé local ont été collectées à Cotonou, la capitale économique et la ville la plus peuplée du Bénin (Institut national de la statistique et de l'analyse économique du Bénin, 2011). Les données sur la production du riz étuvé local ont été collectées dans la commune de Glazoué, commune qui fournit la plus grande partie du riz étuvé local retrouvé sur les marchés étudiés.

\section{4 Échantillonnage}

La méthode non probabiliste des quotas, largement utilisée dans les enquêtes d'opinion et les études de marché, nous a permis de sélectionner les 210 acteurs interrogés.

\subsubsection{Choix des consommateurs}

Selon Rutsaert et al. (2009), les caractéristiques des ménages ont des effets très limités sur leur choix du riz. De ce 


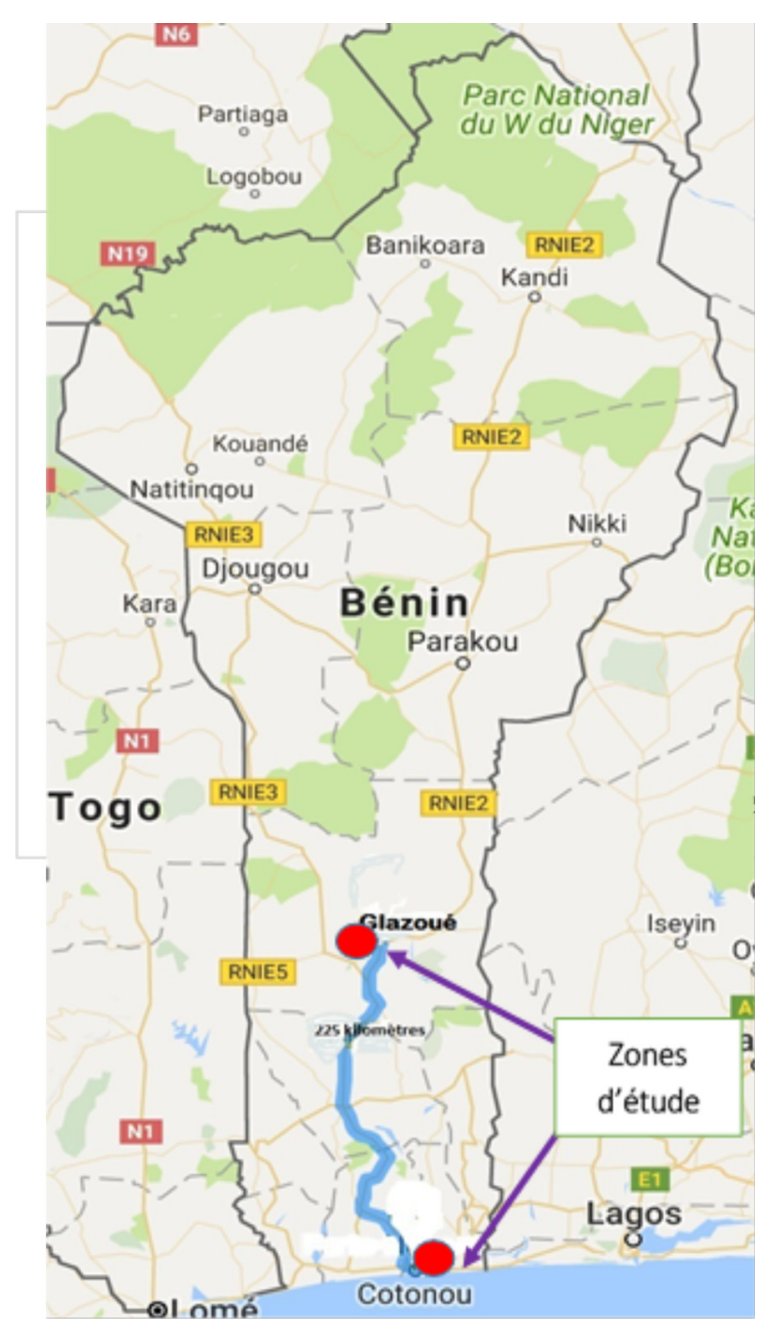

Fig. 1. Zones d'étude.

Fig. 1. Study Areas.

fait, soixante ménages ont été sélectionnés de façon aléatoire dans les deux arrondissements les plus peuplés de Cotonou.

\subsubsection{Choix des commerçants}

Cinquante commerçants ont été choisis sur les trois circuits d'approvisionnement alimentaire utilisés par les ménages à Cotonou:

- le marché de Dantokpa. Situé à trois kilomètres du port de Cotonou, c'est le plus grand marché du Bénin. C'est à la fois un marché de gros, de demi-gros et de détail. Vingtcinq commerçants semi-grossistes et détaillants ont été choisis sur ce marché;

- les marchés de quartier. Essentiellement destinés à la vente au détail, les marchés de quartier fréquentés par les ménages interrogés sont les marchés Ganhi et Saint-Michel, les supermarchés Mont Sinaï et Franc Prix, et la boutique Label Bénin. Cinq commerçants ont été sélectionnés sur ces marchés;

- les circuits de distribution hors des marchés. Ils regroupent la vente de rue fixe, la vente ambulante, la vente permanente à domicile et la vente occasionnelle à domicile. Vingt détaillants ayant des boutiques de vente de riz ont été sélectionnés dans les arrondissements d'enquête.

\subsubsection{Choix du riz étuvé importé}

Le riz étuvé importé le plus populaire à Cotonou (et le plus vendu) est celui choisi pour les analyses comparatives. Il s'agit du riz étuvé de la marque MEME à $10 \%$ de brisures.

\subsubsection{Choix des producteurs, étuveuses}

Les enquêtes réalisées auprès des commerçants de riz étuvé local et des consommateurs ont révélé que la commune de Glazoué fournit $85 \%$ du riz étuvé local retrouvé sur les marchés étudiés. Cinquante producteurs ont été sélectionnés de façon aléatoire dans cette commune. Ces producteurs approvisionnent à la fois les étuveuses et les transformateurs de paddy en riz blanc local; et vendent leur paddy au plus offrant.

Cinquante étuveuses ont été également sélectionnées dans la commune de Glazoué : $50 \%$ de ces étuveuses approvisionnent les marchés urbains et les autres vendent la totalité de leur produit sur les marchés ruraux.

\subsection{Outils d'analyse}

Le test de Kendall a été utilisé pour déterminer la corrélation entre l'indisponibilité du riz étuvé local et les causes identifiées par les commerçants d'une part et les ménages d'autre part. C'est un test non paramétrique de corrélation de rang entre deux variables. Comparé aux tests de corrélation de Spearman et de Pearson, le test de Kendall a l'avantage de transcender la métrique des variables mesurées et d'écarter la nécessité d'un modèle paramétrique (Laurencelle, 2009). Le coefficient de corrélation de Kendall varie de -1 à +1 et est obtenu par la formule :

$$
\tau=\frac{\begin{array}{c}
\text { (nombre de paires concordantes) } \\
\text { (nombre de paires discordantes) }
\end{array}}{\frac{1}{2} * n *(n-1)} .
$$

Lorsque $\tau$ tend vers 1 , alors il y a une corrélation positive entre les variables.

\section{Résultats et discussion}

\subsection{L'insignifiante place du riz étuvé local dans l'offre de riz à Cotonou}

La figure 2 présente l'offre de riz à Cotonou et montre que le riz importé représente $88 \%$ de l'offre totale contre $12 \%$ pour le riz local. Ce pourcentage est un peu supérieur aux résultats donnés par l'étude récente de Demont et al. (2017) qui rapporte que la part de riz importé au Bénin est de $75 \%$. Cette proportion de riz importé à Cotonou peut aussi s'expliquer par la proximité du port, toutes les villes portuaires d'Afrique de l'Ouest ayant une dépendance accrue aux aliments importés selon de nombreux auteurs (par exemple, Bricas et al., 2016).

La figure 2 révèle aussi la prédominance du riz blanc, surtout du riz blanc importé qui représente $73 \%$ de l'offre en riz. Ce résultat corrobore les conclusions des études de Naseem et al. (2013) et Kinkpé et al. (2016) : les Béninois préfèrent le riz blanc importé. 


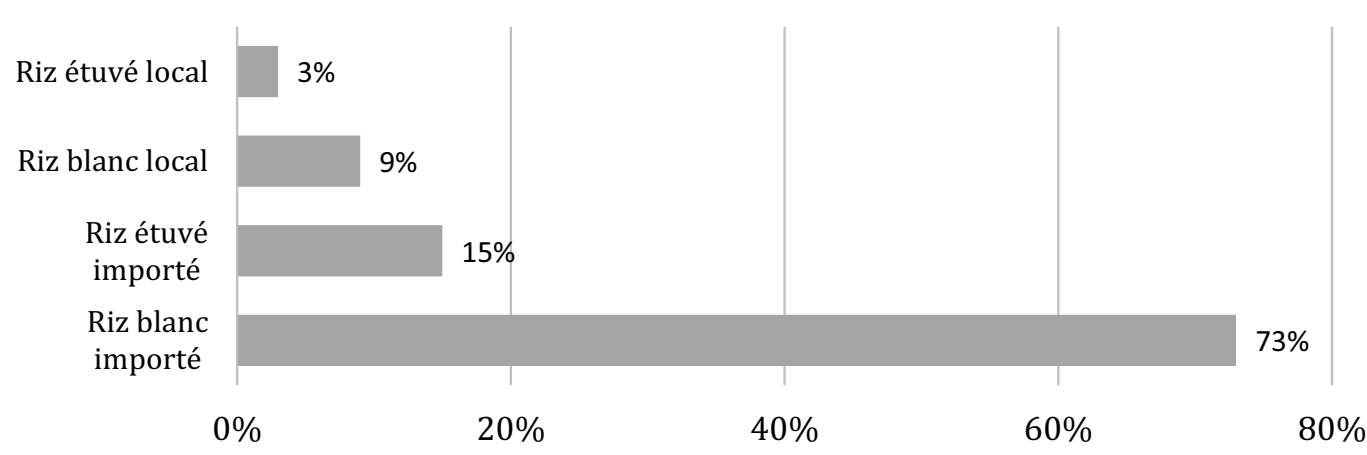

Fig. 2. Part des différents types de riz disponibles sur les marchés de Cotonou.

Fig. 2. Parts of different types of rice available on the Cotonou markets.

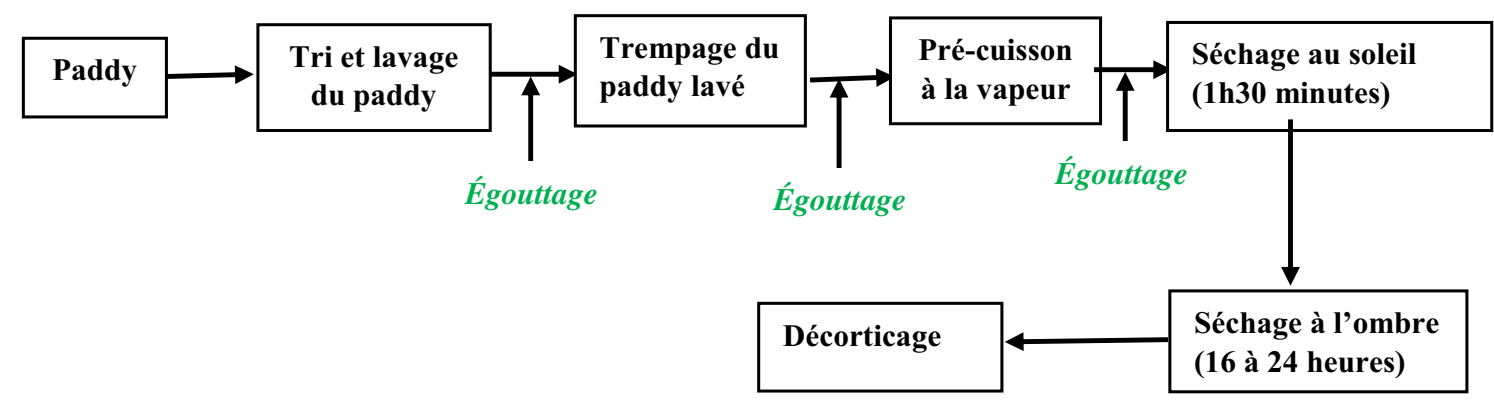

Fig. 3. Processus d'étuvage du paddy.

Fig. 3. Process of parboiling rice in Collines.

En ce qui concerne le riz étuvé local, il ne représente que $3 \%$ de l'offre totale de riz, cinq fois moins que le riz étuvé importé.

\subsection{Commerce du riz étuvé local : acteurs, stratégies d'approvisionnement et formation du prix au consommateur}

\subsubsection{Approvisionnement}

L'analyse de la provenance du riz étuvé local présent sur les marchés étudiés indique que $85 \%$ de ce riz provient du département du Zou-Collines et plus précisément de la commune de Glazoué.

Dans ce département, ce sont les femmes de la commune de Glazoué, commune située à 25 kilomètres de Cotonou, qui fournissent la quasi-totalité du riz étuvé local vendu à Cotonou. Ce résultat s'explique par le fait que l'étuvage est une activité économique très ancienne dans cette commune et par les nombreux appuis techniques et/ou matériels dont ont bénéficié les étuveuses de cette commune depuis la crise rizicole de 2008 (dons des organismes comme SNV, VECO WA, AfricaRice). C'est aussi dans cette commune qu'a vu le jour en 2015 la première union départementale des étuveuses au Bénin : l'Union régionale des femmes étuveuses du riz des Collines (URFER-C).

L'étuvage se fait en sept étapes comme le montre la figure 3.

Le paddy trié est lavé puis transvasé dans un panier propre pour l'égouttage. Il est renversé ensuite dans une marmite contenant de l'eau et mis au feu jusqu'au début de l'ébullition où la marmite est retirée du feu et laissée au repos pour refroidir pendant environ 12 heures.
Le paddy est enlevé de la marmite, lavé et égoutté. Il est ensuite versé dans le bac d'étuvage et cuit à la vapeur, puis séché au soleil et à l'ombre. Pour le décorticage, les étuveuses font appel aux meuniers et le rendement moyen au décorticage est de $64 \%$.

\subsubsection{Structure des marchés du riz étuvé local et comportement des acteurs}

Les acteurs et les circuits de distribution du riz importé et $\mathrm{du}$ riz local (en provenance des Collines) à Cotonou sont présentés par la figure 4.

Cette figure montre que les détaillants sont les seuls acteurs à commercialiser à la fois le riz local et le riz importé. Ils fournissent la quasi-totalité $(90 \%)$ du riz acheté par les ménages. Pendant les périodes de fêtes (fin d'année ou cérémonies), les ménages s'approvisionnent auprès des semigrossistes car les quantités achetées sont supérieures ou égales à $25 \mathrm{~kg}$.

À l'exception de la boutique label Bénin qui ne vend que le riz local (étuvé et blanc), tous les détaillants de riz étuvé local vendent au moins six différents riz importé. Par ailleurs, les commerçants de riz interrogés adoptent la même stratégie que ceux du Ghana en associant la vente du riz à celle d'autres produits alimentaires et non alimentaires (Tomlins et al., 2005).

En ce qui concerne les relations entre les commerçants, aucune stratégie anticoncurrentielle entre les semi-grossistes du riz local et ceux du riz importé n'a été notée pendant nos enquêtes ni rapportée par les commerçants eux-mêmes. 


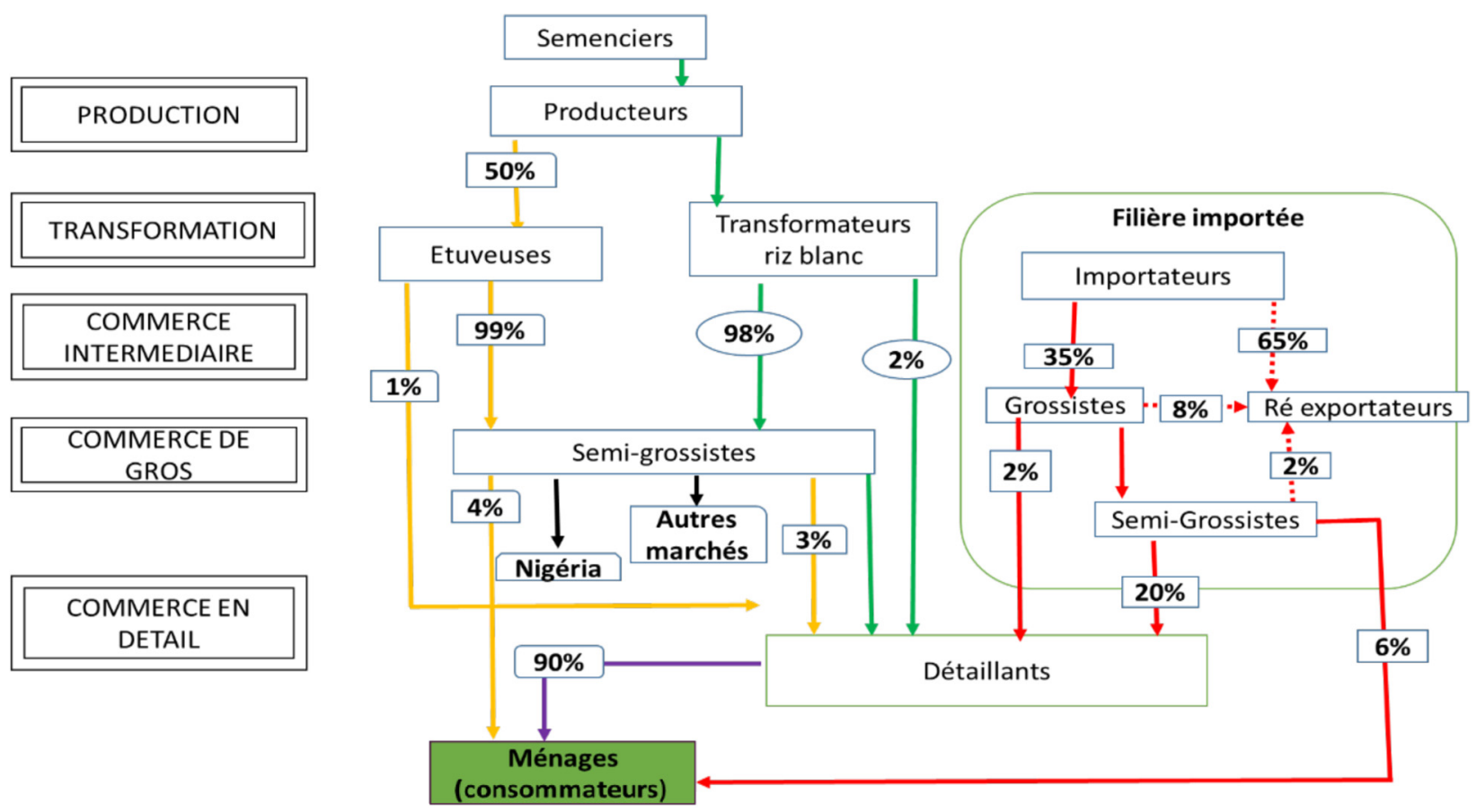

Fig. 4. Circuit de distribution du riz des Collines et du riz importé sur les marchés de Cotonou.

Fig. 4. Circuit of distribution of Collines rice and imported rice on Cotonou's markets.

Les transactions entre acteurs de la filière «importée » sont faites au comptant ou à crédit partiel $(50 \%$ au comptant et solde au prochain achat).

En ce qui concerne le riz local, les transactions entre riziculteurs et étuveuses sont au comptant (65\%), à crédit partiel $(20 \%)$ ou à crédit total (paiement après la vente du riz étuvé : $15 \%)$. Ces crédits sont obtenus auprès des producteurs avec lesquels les étuveuses ont des liens de parenté, qui sont peu nombreux et ne fournissent que de faibles quantités. Les transactions entre les étuveuses et les semi-grossistes sont au comptant ou en préfinancement partiel et les transactions entre semi-grossistes et détaillants sont au comptant. Lorsque les étuveuses ne sont pas préfinancées, le manque de fonds de roulement limite leur capacité d'achat de paddy et augmente le délai de livraison.

\subsubsection{Formation du prix au consommateur du riz étuvé local}

Le tableau 1 présente le compte consolidé de la production et de la commercialisation d'une tonne de riz étuvé local. Il révèle que les consommations intermédiaires hors filière (CIHF) (comme intrants, main-d'œuvre, transport) représentent le poste de dépense le plus élevé. La marge nette est positive pour tous les acteurs, et la marge globale est de $145,6 \mathrm{FCFA} / \mathrm{kg}$ de riz étuvé local ( $1 €=656 \mathrm{FCFA})$. Les étuveuses captent la plus grosse part de cette marge $(28,7 \%)$ et les semi-grossistes, la plus faible part $(21,6 \%)$.

Ce tableau montre également que le RSI de tous les acteurs est supérieur à $7 \%$, taux d'intérêt appliqué à l'épargne par les banques au Bénin.

En ce qui concerne la structure du prix au consommateur du riz étuvé, les CIHF justifient $62 \%$ de ce prix, la marge totale $29 \%$ et les charges fixes $8 \%$.

\subsection{Analyse comparative de la rentabilité du commerce de détail du riz étuvé (local versus importé)}

Le tableau 2 présente une analyse comparative de la rentabilité du commerce au détail du riz étuvé. Il montre que le détaillant achète le riz étuvé local à $450 \mathrm{FCFA} / \mathrm{kg}$ et le riz étuvé importé à 360 FCFA : une différence de 90 FCFA en défaveur du riz étuvé local. Il revend le riz étuvé local à $500 \mathrm{FCFA} / \mathrm{kg}$ et le riz étuvé importé à 400 FCFA. À ces différences de prix, s'ajoute une différence de volume traité : $250 \mathrm{~kg}$ de riz étuvé importé sont vendus chaque mois contre $132 \mathrm{~kg}$ pour le riz étuvé local. Il s'ensuit alors que la $\mathrm{MN}$ mensuelle dégagée par le détaillant sur le riz étuvé importé (5280 FCFA) est supérieure à celle obtenue sur le riz étuvé local (4750 FCFA), bien que la $\mathrm{MN} / \mathrm{kg}$ soit plus intéressante sur le riz étuvé local : 36 FCFA contre 21 FCFA pour le riz étuvé importé.

\subsection{Facteurs limitant la disponibilité du riz étuvé local à Cotonou}

\subsubsection{Facteurs limitant selon les commerçants}

Selon les commerçants, la méconnaissance des valeurs nutritives du riz étuvé local par les ménages, la mévente et l'indisponibilité du riz local sont les trois principaux facteurs limitant sa disponibilité à Cotonou (coefficient de Kendall, $\tau=0,927$ et chi carré, $\left.\chi^{2}=278,147\right)$.

\subsubsection{Facteurs limitant selon les ménages}

Les ménages identifient également la méconnaissance de ce riz et de ses valeurs nutritionnelles par les consommateurs comme premier facteur limitant sa commercialisation. 
Tableau 1. Compte consolidé de la production d'une tonne de riz étuvé dans les Collines.

Table 1. Consolidated account of the production of one ton of parboiled rice in the Collines.

\begin{tabular}{|c|c|c|c|c|c|c|}
\hline & Producteurs & Étuveuses & Semi-grossistes & Détaillants & Total & $\begin{array}{l}\text { Part dans le prix } \\
\text { de vente du riz }(\%)\end{array}$ \\
\hline Coûts fixes (FCFA) & 6549 & 22933 & 5366 & 6057 & 40905 & 8,18 \\
\hline Coût des CIHF (FCFA) & 197140 & 95220 & 11480 & 7000 & 310840 & 62,18 \\
\hline Autres coûts (FCFA) & 0 & - & 1640 & 1000 & 2640 & 0,5 \\
\hline Coût total production (FCFA) & 203688 & 358153 & 418486 & 464056 & 354384 & - \\
\hline $\mathrm{MN}$ en filière (FCFA) & 36312 & 41847 & 31514 & 35943 & 145616 & 29,12 \\
\hline MN de l'acteur/ MN totale (\%) & 24,9 & 28,7 & 21,6 & 24,7 & 1 & - \\
\hline RSI & 17,82 & 11,68 & 7,53 & 7,74 & & - \\
\hline
\end{tabular}

Tableau 2. Analyse comparative de la rentabilité mensuelle de la vente au détail du riz étuvé importé et du riz étuvé local.

Table 2. Comparative analysis of the monthly retail profitability of imported parboiled rice and local parboiled rice.

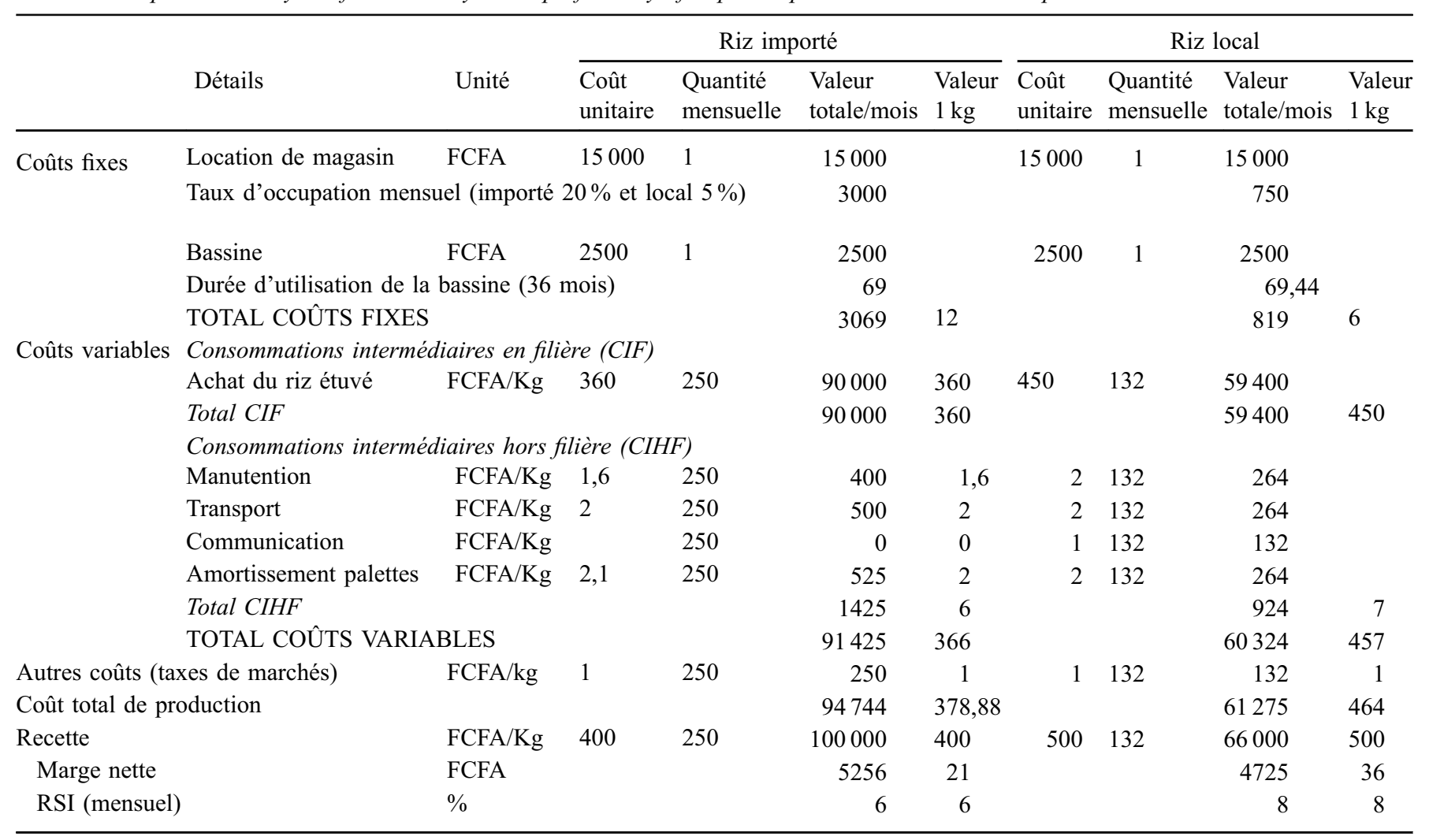

Dans notre échantillon, plus de $90 \%$ des ménages ne connaissaient pas le riz étuvé local (55 sur les 60). Le second facteur limitant est la différence de prix de $100 \mathrm{FCFA} / \mathrm{kg}$ en défaveur du riz étuvé local alors que les consommateurs ne notent aucune différence physique entre les deux riz. L'indisponibilité du riz étuvé auprès de tous les commerçants et les fréquentes ruptures de stock constituent, respectivement, les troisième et quatrième facteurs limitants.

Le temps de cuisson plus long que celui du riz étuvé importé et sa texture dure occupent la cinquième et la sixième position. Les ménages affirment en effet que lorsque le riz étuvé local préparé refroidit, sa texture est plus dure que celle du riz étuvé importé.

Le coefficient de Kendall obtenu à ce niveau est $\tau=0,681$ (et chi carré, $\chi^{2}=274,532$ ).

Pour ce qui est de la propreté, du taux de brisure et de la capacité de gonflement, les ménages avouent que le riz étuvé local est identique au riz étuvé importé. Les efforts mobilisés depuis 2008 ont apparemment permis aux étuveuses de corriger ces défauts, qui étaient attribués au riz étuvé local en 2008 (Demont et al., 2013). 


\section{Conclusion}

Le riz étuvé local, par ses valeurs nutritionnelles meilleures que celles du riz blanc, est une alternative intéressante pour infléchir la dépendance du Bénin au riz importé. Depuis 2008, des efforts ont été consentis pour augmenter la production et améliorer la qualité du riz à travers la mise au point de kits modernes d'étuvage. Malgré ces efforts, l'offre du riz étuvé local est encore très limitée, surtout pour les marchés urbains, plus rémunérateurs que les marchés ruraux mais plus dépendants au riz importé. Nos résultats indiquent que le problème du riz étuvé local est à la fois lié aux habitudes alimentaires des ménages et à l'irrégularité de l'offre des étuveuses. Mais avec des sensibilisations sur les valeurs nutritionnelles du riz étuvé local, les consommateurs urbains pourraient modifier leur comportement en faveur de ce riz.

Nos résultats confirment ainsi que la dépendance urbaine au riz importé peut être infléchie à condition que la population urbaine soit sensibilisée sur les valeurs nutritives du riz étuvé local et que les étuveuses travaillent à améliorer leur offre.

Le riz étuvé local peut donc être une alternative intéressante pour les pays d'Afrique de l'Ouest qui ont misé depuis 2008, sans succès, sur leur riz blanc local pour réduire leur dépendance au riz importé.

Toutefois, étant donné que le riz étuvé est également importé, nous recommandons que des études sur les critères de choix du riz étuvé par les ménages soient conduites afin de permettre aux étuveuses de proposer un riz étuvé local qui réponde aux attentes des consommateurs.

\section{Références}

Adegbidi A. 1996. Structure, conduite et performance du marché du porc local au Sud-Bénin. Cotonou: Faculté des Sciences Agronomique-Université National du Bénin, Série d'Économie et de Sociologie Rurale $\mathrm{n}^{\mathrm{o}} 20$.

Adégbola P, Midingoyi S-K, Djenontin I, Arouna A, Adekambi S. 2011. Analyse de la performance des chaînes de valeur Riz au Bénin. Cotonou: PAPA/INRAB/MAEP et ADRAO.

Bricas N, Tchamda C, Mouton F, eds. 2016. L'Afrique à la conquête de son marché alimentaire intérieur. Enseignements de dix ans d'enquête auprès des ménages d'Afrique de l'ouest, au Cameroun et du Tchad. Paris : AFD, «Études de l'AFD» $\mathrm{n}^{\circ} 12$.

Dabat M-H, Lançon F, Hanak H, Fabre P. 2007. Manuel d'analyse des filières agroalimentaires. Montpellier: CIRAD.

Demont M, Ndour M, Zossou E. 2013. Le riz africain peut-il être compétitif? Une analyse de la compétitivité-qualité par la méthode des enchères expérimentales. Cahiers Agricultures 22: 345-352. DOI: 10.1684/agr.2013.0664.

Demont M, Fiamohé R, Kinkpé T. 2017. Comparative advantage in demand and the development of rice value chains in West Africa. World Development 96: 578-590. DOI: 10.1016/j.world dev.2017.04.004.

Gounsè. 2006. Analyse socio-économique de la commercialisation du riz local au Centre-Bénin. Thèse d'Ingénieur Agronome. Faculté des Sciences Agronomiques de l'Université d'Abomey-Calavi, Bénin.
Hatcheu. 2003. L'approvisionnement et la distribution alimentaires à douala (Cameroun) : logiques sociales et pratiques spatiales des acteurs. Thèse de Doctorat en géographie, Université de Paris I; IRD. Disponible sur http://www.cartographie.ird.fr/publi/Hatcheu. pdf.

Houssou P, Gankoue B, Kaboré A, Futakuchi K, Traoré K, Moreira J, et al. 2016. Comparison of parboiled and white rice obtained from ten varieties cultivated in Benin. International Food Research Journal 23(6): 2479-2486.

Institut national de la statistique et de l'analyse économique du Bénin. 2011. Rapport final de l'enquête modulaire intégrée sur les conditions de vie des ménages. Disponible sur http://www.insae-bj. org/emicov.html.

Kinkpé T, Adegbola P, Yabi J, Adekamni S, Biaou G. 2016. Analyse conjointe de la préférence des consommateurs pour les attributs de marche du riz au Benin. 5th International Conference of the African Association of Agricultural Economists, September 23-26, 2016, Addis Ababa.

Konnon D-D, Sotondji C, Adidéhou A 2014. État des lieux de la filière riz au Bénin en 2014. Rapport d'étude. Conseil de concertation des riziculteurs du Bénin. Disponible sur www. interreseaux.org/IMG/pdf/Rapport_Final_Etat_des_Lieux Riz_1_.pdf.

Lançon F. 2011. La compétitivité du riz ouest-africain face aux importations: vrais enjeux et fausses questions. Grain de $\mathrm{Sel}$ 54-56: 21-22. Disponible sur http://www.inter-reseaux.org/IMG/ pdf/GDS54-56_Cereales.pdf.

Laurencelle L. 2009. Le tau et le tau-b de Kendall pour la corrélation de variables ordinales simples ou catégorielles. Tutorials in Quantitative Methods for Psychology 5(2): 51-58. DOI: 10.20982/ tqmp.05.2.p052.

Lutz C 1994. The functioning of the maize market in Benin: spatial and temporal arbitrage on the market of a staple food crop. $\mathrm{PhD}$ thesis. Department of Regional Economics, University of Amsterdam.

Naseem A, Mhlanga S, Diagne A, Adegbola P, Midingoyi S-K. 2013. Economic analysis of consumer choices based on rice attributes in the food markets of West Africa-the case of Benin. Food Security 5(4): 575-589. DOI: 10.1007/s12571-013-0276-6.

Ndindeng S, Manful J, Futakuchi K, Mapiemfu-Lamare D, AkoaEtoa J, Tang E, et al. 2015. Upgrading the quality of Africa's rice: A novel artisanal parboiling technology for rice processors in Sub-Saharan Africa. Food Science \& Nutrition 3(6): 557-568. DOI: $10.1002 / \mathrm{fsn} 3.242$.

Olagunju F, Babatunde R, Salimonu K. 2012. Market structure, conduct and performance of gari processing industry in South Western Nigeria. European Journal of Business and Management 4: 99-112. Available from http://www.iiste.org/Journals/index. php/EJBM/article/viewFile/1039/959.

Rutsaert P, Demont M, Ndour M, Tollens E 2009. Competitive rivals: Willingness to pay for Senegal river valley versus imported rice. 2nd EAAE Workshop on valuation methods in agro-food and environmental economics experimental auction: Theoretical background and empirical applications. Barcelona.

Tomlins K, Manful J, Larwe P, Hammond L. 2005. Urban consumer preferences and sensory evaluation of locally produced and imported rice in West Africa. Food Quality and Preference 16(1): 79-89. DOI: 10.1016/j.foodqual.2004.02.002.

Citation de l'article : Todomé L, Lejars C, Lançon F, Hamimaz R. 2018. Pourquoi le riz étuvé local est-il peu disponible sur les marchés urbains du Bénin? Cah. Agric. 27: 15009. 